\title{
Gas Sensors Based on Layer-By-Layer Assembled Graphene Oxide and Reduced Graphene Oxide
}

\author{
Jia-Jia SONG ${ }^{1,2}$, Yan-Yan WANG ${ }^{1,2, a^{*}}$, Ming YANG $^{1,2}$, Feng ZHANG ${ }^{1,2}$, \\ Yan YE ${ }^{1,2}$, Yan-Hua LIU ${ }^{1,2}$, Lin-Sen CHEN ${ }^{1,2}$, Chang-Si PENG ${ }^{1,2, b^{*}}$ \\ ${ }^{1}$ College of Physics, Optoelectronics and Energy \& Collaborative Innovation Center of Suzhou \\ Nano Science and Technology, Soochow University, Suzhou 215006, China \\ ${ }^{2}$ Key Lab of Advanced Optical Manufacturing Technologies of Jiangsu Province \& Key Lab of \\ Modern Optical Technologies of Education Ministry of China, Soochow University, Suzhou 215006, \\ China \\ ayywang@suda.edu.cn, bchangsipeng@suda.edu.cn \\ ${ }^{*}$ Corresponding author
}

Keywords: Layer-By-Layer, Graphene Oxide, Reduced Graphene Oxide, Gas Sensor.

\begin{abstract}
Based on graphene oxide and reduced graphene oxide, a novel gas sensor was fabricated with layer-by-layer self-assembly techniques. The negatively charged graphene oxide sheets and the positively charged reduced graphene oxide sheets were layer-by-layer assembled on positive Au electrodes. The gas sensor exhibited a $1.83 \%$ of resistance difference before and after exposure to $20 \mathrm{ppm}$ of dimethyl methylphosphonate (DMMP). The sensor showed a good repeatability, and the recovery time was about 4 min. Above all, the unique properties, such as simplicity of production process, the easy availability of the materials and the good response to DMMP vapor, endow the device a great potential for sensing application.
\end{abstract}

\section{Introduction}

Gas sensor becomes more and more important in numerous fields, such as environmental monitoring, industry, medicine, energy and public safety[1-5]. Nanoscale materials, including semiconductor nanowires, carbon nanotubes and graphene, are considered as promising candidates for high performance gas sensor due to their small size, large specific surface area as well as low cost [6-8]. Carbon nanotubes and graphene have been widely reported on monitoring toxic gas, including $\mathrm{CO}, \mathrm{CO}_{2}, \mathrm{NH}_{3}, \mathrm{NO}, \mathrm{NO}_{2}$ [9-13]. However, it is still a challenge to develop portable gas sensors with high selectivity, excellent stability at room temperature.

Graphene, as a single atomic layer 2-dimensional honeycomb carbon sheet[12] has aroused a great attention, due to its miracle properties, including high carrier mobility[13], large surface areas[14], high specific transparency[15], exceptional thermal conductivity[16], as well as high Young's modulus[17]. Graphene is a single layer 2D flat material. The specific surface areas reach up to $2630 \mathrm{~m}^{2} / \mathrm{g}$. All carbon atoms can be exposed to the environment, which leading to great adsorption of gas. Gas sensor based on graphene shows high sensitivity to the gas molecules[18]. The signal-to-noise ratio of graphene gas sensor is very high due to the low electrical noise and high carrier mobility of graphene.

Many techniqueshave been reported to prepare graphene, including micromechanical exfoliation[12], chemical vapor decomposition[19], epitaxial growth[20], chemical or thermal reduction of graphene oxide[21]. Among them, the chemically reduced graphene oxide (rGO) is considered as an excellent material for gas sensing due to its facile solution process, low cost, scalable production, and large available surface areas[22-26]. On the surface of rGO, there are full of defects and chemical groups can enhance gas adsorption. 
In this paper, we report a novel gas sensor based on layer-by-layer (LbL) self-assembled GO and rGO on the Au electrodes. The negative of GO assembles on the positive charged Au electrodes when modified by 3-Aminopropyltrimethoxysilane (APS), then the positive rGO assembles on the surface of negative GO.

\section{Experiment}

\section{Preparation of the Go and Rgo}

The negatively charged GO was prepared by a modified Hummers method[27]. The positively charged rGO was prepared via the reduction of GO by p-phenylenediamine (PPD). In detail, 650 $\mathrm{mg}$ PPD was add to $75 \mathrm{ml}$ alcohol, afterwards, $150 \mathrm{ml}$ of $\mathrm{GO}(1 \mathrm{mg} / \mathrm{ml})$ was dispersed in the PPD solution. The mixture was ultrasonicated for a few minutes. The mixture was refluxed in an oil bath at $90{ }^{\circ} \mathrm{C}$ for $16 \mathrm{~h}$ in order to reduce the GO. Then the black mixture was filtered and washed with DMF, water, alcohol three times in sequence. Subsequently, the mixture was diluted with alcohol in order to obtain the suspensions with different concentrations.

\section{Lbl Self-Assembly of Go and Rgo on the Au Electrodes}

In order to get the positively charged interdigital electrode, the clean electrodes were immersed in the mixture of $\mathrm{H}_{2} \mathrm{SO}_{4}: \mathrm{H}_{2} \mathrm{O}_{2}(3: 1)$ at $90{ }^{\circ} \mathrm{C}$ for $1 \mathrm{~h}$. Then the electrode was washed with distilled water, followed by immersing in APS solution for $3 \mathrm{~h}$. The electrodes were then put in oven at $150{ }^{\circ} \mathrm{C}$ for $3 \mathrm{~h}$. Finally, the electrodes decorated with $-\mathrm{NH}_{2}$ were obtained. The positively charged electrodes were firstly immersed in GO solution for $20 \mathrm{~min}$ to render the electrode negative charged, then it's cleaned by water and alcohol separately. The electrode was then transferred to the positive charged rGO solution for $20 \mathrm{~min}$, subsequently washed by alcohol and water separately. This process was repeated for required cycles to achieve the $(\mathrm{GO} / \mathrm{rGO}) \mathrm{n}$ sensor.

\section{Characterization}

National Instruments 380ZLS was used to evaluate the Zeta potential of GO and rGO solution. Hitachi SU8010 was used for the scanning electron microscopy(SEM) image. A homemade test system was designed to detect the performance of the LbL self-assembled (GO/rGO)n sensor which was mentioned in the previous studies[28].

\section{Results and Discussion}

\section{(Go/Rgo)N Materials}

The potential of GO and $\mathrm{rGO}$ solutions $(1 \mathrm{mg} / \mathrm{ml})$ were tested by zeta potential analyzer. The potential of $\mathrm{GO}$ was $-20.80 \mathrm{mV}$ and that of $\mathrm{rGO}$ was $0.15 \mathrm{mV}$, which indicated that the negatively charged GO and the positively charged rGO were successfully achieved.

The surface topography of the $(\mathrm{GO} / \mathrm{rGO}) \mathrm{n}$ materials with different layer number $\mathrm{n}$ was evaluated by SEM (Fig. 1). From the SEM images, it is observed that $(\mathrm{GO} / \mathrm{rGO}) \mathrm{n}$ were successfully assembled on the electrode by electrostatic attraction. 


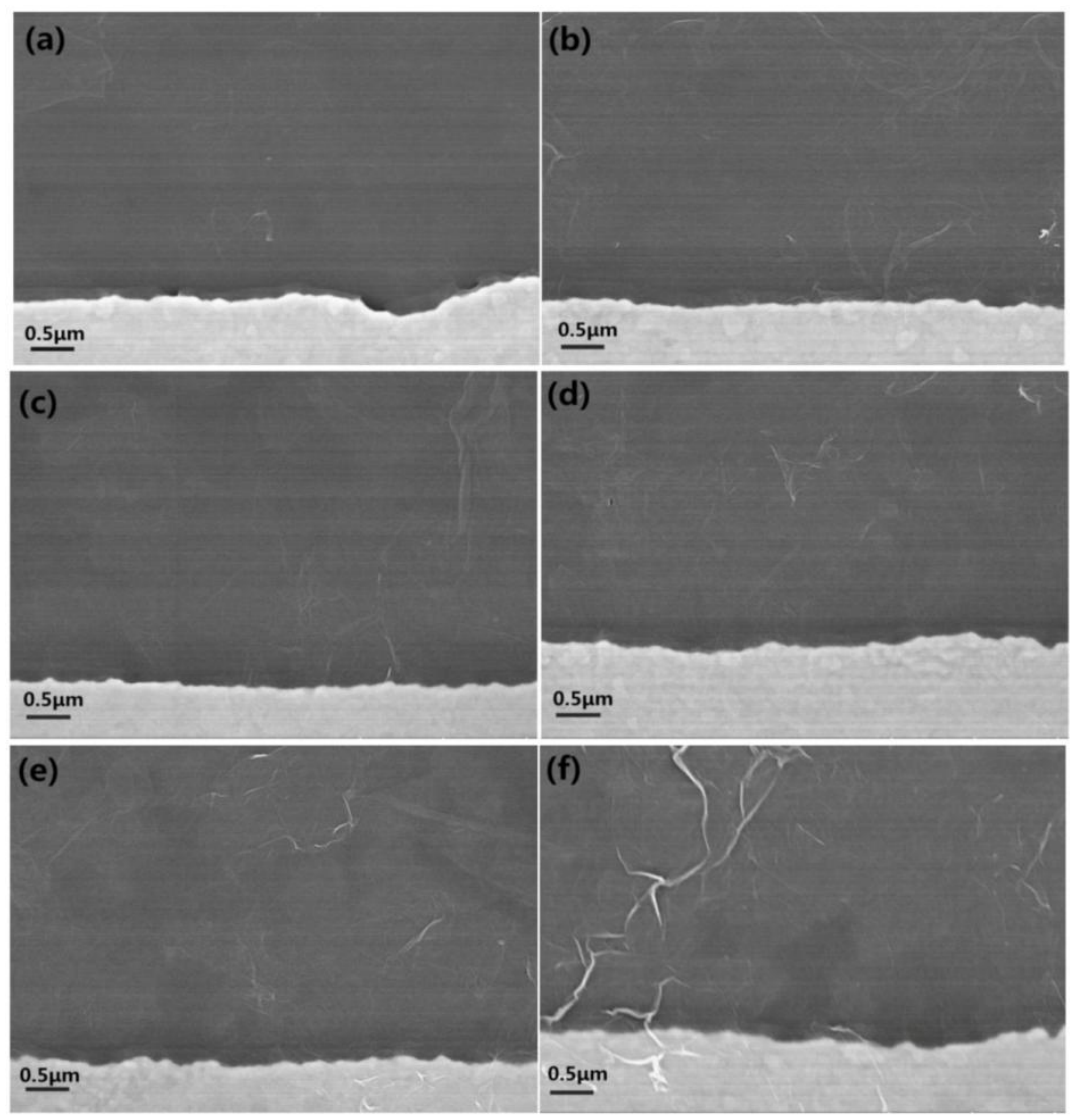

Fig. 1 SEM images of (a) (GO/rGO)2; (b) (GO/rGO)4; (c) (GO/rGO)6; (d) (GO/rGO)8; (e) $(\mathrm{GO} / \mathrm{rGO}) 10$; and (f) $(\mathrm{GO} / \mathrm{rGO}) 12$

\section{Gas Sensor}

The sensor performances of $(\mathrm{GO} / \mathrm{rGO}) \mathrm{n}$ gas sensors based on assembled $\mathrm{GO}$ and $\mathrm{rGO}$ were analyzed as shown in Fig. 2-5. Once the sensor was exposed to the DMMP, it responded quickly. For different concentrations of DMMP, the response of the $(\mathrm{GO} / \mathrm{rGO}) n$ sensor was obtained by the following equation ${ }^{[29]}$ :

$$
\mathrm{R}(\%)=100 \times\left(\mathrm{R}_{\mathrm{DMMP}}-\mathrm{R}_{0}\right) / \mathrm{R}_{0}
$$

Where, $R_{0}$ and $R_{D M M P}$ are the resistance of sensors before and after exposure of the device to DMMP.

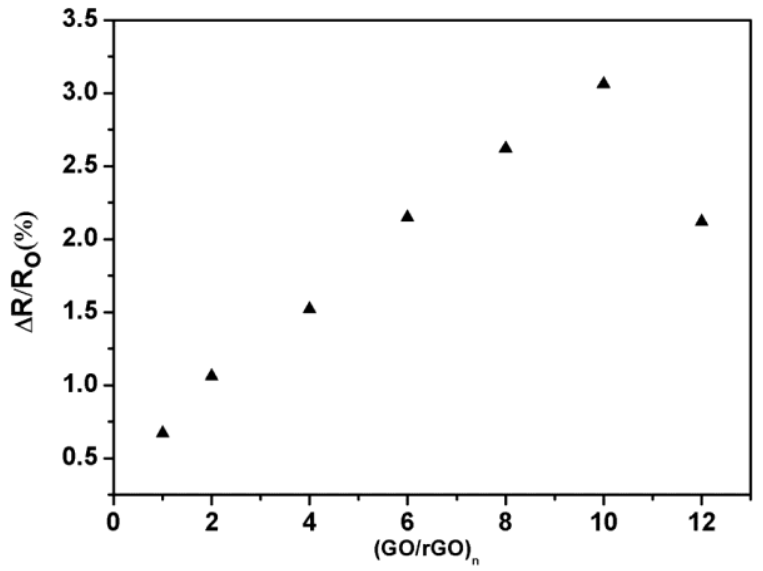

Fig. 2 Response of (GO/rGO)n sensor with layer number for 50 ppm of DMMP 
Fig. 2 demonstrated that the sensor prepared with different layers of GO/rGO showed different responses under $50 \mathrm{ppm}$ DMMP. Obviously, with the increase of layers, the response of the sensor also increased. The response of $(\mathrm{GO} / \mathrm{rGO})_{10}$ sensor was reached up to $3.06 \%$. However, when layers of $\mathrm{GO} / \mathrm{rGO}$ was more than 10 , the response was reduced $\left(2.12 \%\right.$ for $\left.(\mathrm{GO} / \mathrm{rGO})_{12}\right)$, probably due to an excess of graphene assembled on the electrode.

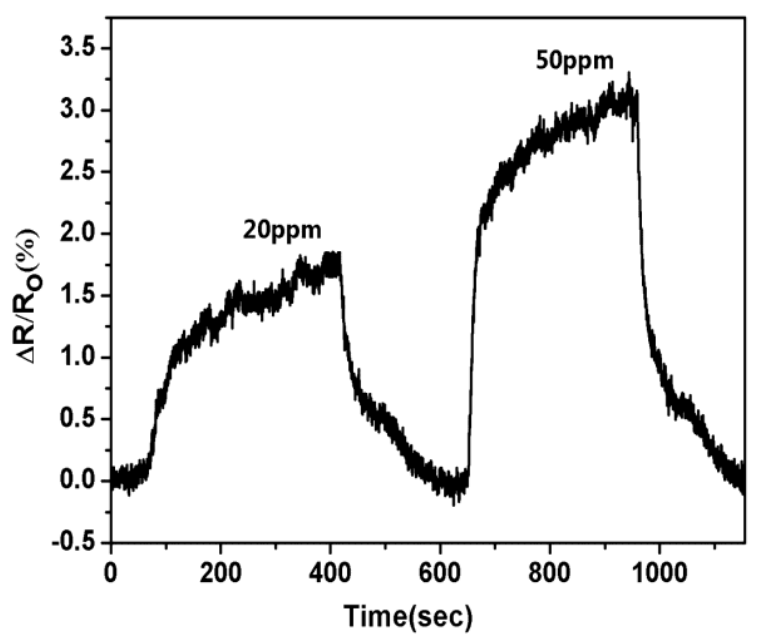

Fig. 3 Response curve of the (GO/rGO)10 sensor for different concentrations of DMMP

10 layers of GO/rGO were used for devices of gas sensor. Fig. 3 showed the $(\mathrm{GO} / \mathrm{rGO})_{10}$ gas sensor for 20 and $50 \mathrm{ppm}$ of DMMP. The response value of the sensor increased with the concentration of DMMP. A $1.83 \%$ resistance variation was achieved when the concentration was as low as 20 ppm. Fig. 3 also showed good recovery performance with recovery time of 4 min.

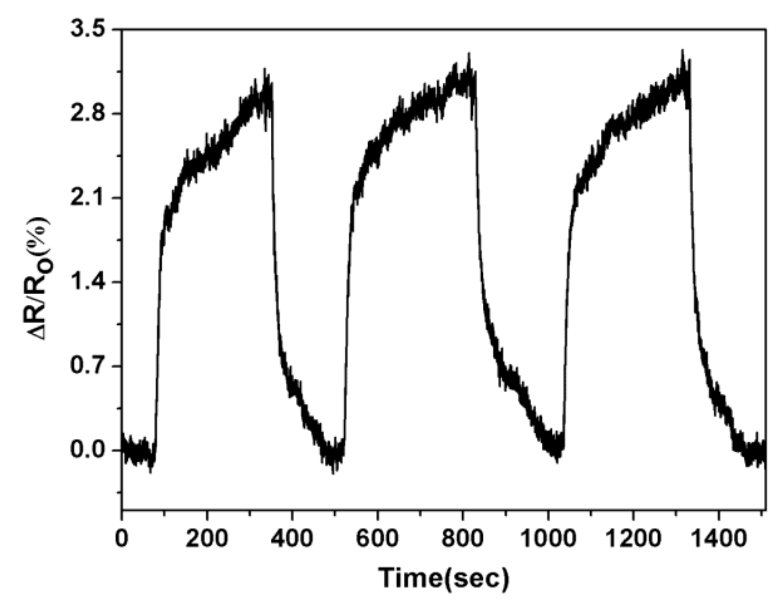

Fig. 4 Repeatability of the (GO/rGO) 10 sensor for 50 ppm DMMP.

Three adsorption and desorption cycles were carried out for testing the repeatability of the sensors. Fig. 4 showed that the resistance of the device increased rapidly at room temperature as soon as the sensor for $50 \mathrm{ppm}$ DMMP. The sensor could recover within $4 \mathrm{~min}$. The three successful adsorption and desorption showed good repeatability.

\section{Conclusions}

A novel sensor based on self-assembled GO and rGO alternative layers was reported for gas sensor. The negatively charged GO and the positive charged rGO was successfully assembled on the positive charged electrode in turn by electrostatic attractions. The effects of different layers of $(\mathrm{GO} / \mathrm{rGO}) \mathrm{n}$ on the gas sensing properties were studied. The results showed that the response of the $(\mathrm{GO} / \mathrm{rGO})_{10}$ sensor exposed to $20 \mathrm{ppm}$ DMMP vapor showed the response as high as $1.83 \%$. Also, the $(\mathrm{GO} / \mathrm{rGO})_{10}$ sensor had a good repeatability and short recovery time for DMMP. 


\section{Acknowledgments}

The authors gratefully acknowledge financial supports by the National Natural Science Foundation of China (no. 51302179, 61575133 and 11504251), the Natural Science Foundation of the Jiangsu Higher Education Institutions of China (no. 13KJB430018), the Natural Science Foundation of Jiangsu Province (no.BK2012184), the Suzhou Sci-tech Development Project (no.ZXG2013040), the Specialized Research Fund for the Doctoral Program of Higher Education (no. 20133201120027), the International Cooperation Projects (2014DFG12600) by MOST, the Priority Academic Program Development (PAPD) of Jiangsu Higher Education Institutions.

\section{References}

[1] X.L. Huang, N.T. Hu, R.G. Gao, Y. Yu, Reduced graphene oxide-polyaniline hybrid: preparation, characterization and its applications for ammonia gas sensing, Journal of Materials Chemistry. 22 (2012) 22488-22495.

[2] N.T. Hu, Y.Y. Wang, J. Chai, R.G. Gao, Z. Yang, E.S.W. Kong, Y.F. Zhang, Gas sensor based on p-phenylenediamine reduced graphene oxide, Sensors and Actuators B: Chemical. 163 (2012) 107-114.

[3] X. Liu, S.T. Cheng, H. Liu, S. Hu, A survey on gas sensing technology, Sensors. 12 (2012) 9635-9665.

[4] T. Kuila, S. Bose, P. Khanra, A.K. Mishra, N.M. Kim, J.H. Lee, Recent advances in graphenebased biosensors, Biosensors and Bioelectronics. 26 (2011) 4637-4648.

[5] R.K. Joshi, H. Gomez, F. Alvi, A. Kumar, Graphene films and ribbons for sensing of O2, and 100 ppm of CO and NO2 in Practical Conditions, The Journal of Physical Chemistry C. 114 (2010) 6610-6613.

[6] Y. Cui, Q.Q. Wei, H.K. Park, C.M. Lieber, Nanowire nanosensors for highly sen-sitive and selective detection of biological and chemical species, Science. 293 (2001) 1289-1292.

[7] Y.Y. Wang, Y.H. Liu, L. Yuan,F. Zhang, C. Rong, D.Y. Huo, X.Y.Zhang, S.P. Chang, Gas sensors based on deposited single-walled carbon nanotubes-polypyrrole networks for ammonia detection, Advanced Materials Research. 815 (2013) 501-507.

[8] K. Toda, R. Furue, S. Hayami, Recent progress in applications of graphene oxide for gas sensing: a review, Analytica chimica acta. 878 (2015) 43-53.

[9] D.R. Kauffman, D.C. Sorescu, D.P. Schofield, B.L. Allen, K.D. Jordan, A. Star, Understanding the sensor response of metal-decorated carbon nanotubes, Nano letters. 10 (2010) 958-963.

[10]J.J. Zhao, A. Buldum, J. Han, J.P. Lu, Gas molecule adsorption in carbon nanotubes and nanotube bundles, Nanotechnology.13 (2002) 195-200.

[11]I. Jung, D. Dikin, S. Park, W.W. Cai, S.L. Mielke, R.S. Suoff, Effect of water vapor on electrical properties of individual reduced graphene oxide sheets, The Journal of Physical Chemistry C. 112 (2008) 20264-20268.

[12] K.S. Novoselov, A.K. Geim, S.V. Morozov, D. Jiang, Y. Zhang, S.V. Dubonos, I.V. Grigorieva, A.A. Firsov, Electric field effect in atomically thin carbon films, science. 306 (2004) 666-669.

[13] M. Acik, Y.J. Chabal, Nature of graphene edges: a review, Japanese Journal of Applied Physics. 50 (2011) 070101.

[14]M.D. Stoller, S. Park, Y.W. Zhu, J. An, R.S. Ruoff, Graphene-based ultracapacitors, Nano letters.8 (2008) 3498-3502. 
[15]R.R. Nair, P. Blake, A.N. Grigorenko, K.S. Novoselov, T.J. Booth, T. Stauber, N.M.R. Peres, A.K. Geim, Fine structure constant defines visual transparency of grapheme, Science. 320 (2008) 1308-1308.

[16] S. Ghosh, I. Calizo, D. Teweldebrhan, E.P. Pokatilov, D.L. Nika, A.A. Balandin, W. Bao, F. Miao, C.N. Lau, Extremely high thermal conductivity of graphene: Prospects for thermal management applications in silicon nanoelectronics, IEEE. 2008 1-2.

[17]C. Lee, X.D. Wei, J.W. Kysar, J. Hone, Measurement of the elastic properties and intrinsic strength of monolayer grapheme, science. 321 (2008) 385-388.

[18]F. Schedin, A.K. Geim, Morozov S V, et al. Detection of individual gas molecules adsorbed on graphene[J]. Nature materials, 2007, 6(9): 652-655.

[19] S. Bhaviripudi, X.T. Jia, M.S. Dresselhaus, J. Kong, Role of kinetic factors in chemical vapor deposition synthesis of uniform large area graphene using copper catalyst, Nano letters. 10 (2010) 4128-4133.

[20]C. Berger, Z.M. Song, X.B. Li, X.S. Wu, N. Brown, C. Naud, D. Mayou, T.B. Li, J. Hass, A. N. Marchenkov, E. H. Conrad, P. N. First, W. A. Heer, Electronic confinement and coherence in patterned epitaxial grapheme, Science. 312 (2006) 1191-1196.

[21]Y.Y. Wang, L.L. Zhang, N.T. Hu, Y. Wang, Y.F. Zhang, Z.H. Zhou, Y.H. Liu, S. Shen, C.S. Peng, Ammonia gas sensors based on chemically reduced graphene oxide sheets self-assembled on Au electrodes, Nanoscale research letters. 9 (2014) 1-12.

[22] S.S. Varghese, S. Lonkar, K.K. Singh, S. Swaminathan, A. Abdala, Recent advances in graphene based gas sensors, Sensors and Actuators B: Chemical. 218 (2015) 160-183.

[23] S. Mao, S.M. Cui, G.H. Lu, K. Yu, Z.H. Wen, J. H. Chen, Tuning gas-sensing properties of reduced graphene oxide using tin oxide nanocrystals, Journal of Materials Chemistry. 22 (2012) 11009-11013.

[24] S. Liu, B. Yu, H. Zhang, T. Feng, T. Zhang, Enhancing NO2 gas sensing performances at room temperature based on reduced graphene oxide- $\mathrm{ZnO}$ nanoparticles hybrids, Sensors and Actuators B: Chemical. 202 (2014) 272-278.

[25]Z.B. Ye, Y.D. Jiang, H.L. Tai, Z. Yuan, The investigation of reduced graphene oxide/P3HT composite films for ammonia detection, Integrated Ferroelectrics. 154 (2014) 73-81.

[26] G.H. Lu, S.J. Park, K.H. Yu, R.S. Ruoff, L.E. Ocola, D. Rosenmann,J.H. Chen, Toward practical gas sensing with highly reduced graphene oxide: a new signal processing method to circumvent run-to-run and device-to-device variations, ACS nano. 5 (2011) 1154-1164.

[27] Q. Li, Y.Y. Wang, C. Rong, F. Zhang, Y.H. Liu, L.S. Chen, Q.H. Wang, C.S. Peng, Facile assembly of graphene and titania on micro-structured substrates for superhydrophobic surfaces, Ceramics International. 42 (2016) 2829-2835.

[28] X.L. Huang, N.T. Hu, L.L. Zhang, L.M. Wei, H. Wei, Y.F. Zhang, The NH3 sensing properties of gas sensors based on aniline reduced graphene oxide, Synthetic Metals. 185 (2013) 25-30.

[29]N.T. Hu, Z. Yang, Y.Y. Wang, L.L. Zhang, Y. Wang, X.L. Huang, H. Wei, L.M. Wei, Y.F. Zhang, Ultrafast and sensitive room temperature NH3 gas sensors based on chemically reduced graphene oxide, Nanotechnology. 25 (2013) 025502. 